Ophthalmologe 2021 $118: 186-189$ https://doi.org/10.1007/s00347-020-01130-5 Online publiziert: 3. Juni 2020

(c) Der/die Autor(en) 2020
Loïc Hamon 1 (D) - Elias Flockerzi' · Navid Ardjomand ${ }^{2}$ - Berthold Seitz' · Loay Daas'

${ }^{1}$ Klinik für Augenheilkunde, Universitätsklinikum des Saarlandes (UKS), Homburg/Saar, Deutschland

${ }^{2}$ Sehzentrum für Augenlaser und Augenchirurgie, Graz, Österreich

\title{
Ein 27-jähriger Mann mit „Hornhauttrübung" nach Astverletzung
}

\section{Anamnese}

Ein 27-jähriger Patient stellte sich zum ersten Mal in unserer Sprechstunde zur Mitbeurteilung bei scharf begrenzter „Hornhauttrübung“ am linken Auge vor. Der Patient gab an, dass er vor ca. 20 Tagen beim Wandern einen Baumast in das linke Auge bekommen habe. Seitdem bemerke er eine zunehmende Visusminderung am betroffenen linken Auge. Bei persistierenden Beschwerden konsultierte er seinen Augenarzt, der ihn bei anscheinender Inaktivität der „Trübung“ zur Mitbeurteilung überwies.

Am Tag der Vorstellung berichtete der Patient sowohl über eine persistierende und zunehmende Visusminderung als auch Photophobie am betroffenen linken
Auge. Er litt unter keinen Augenschmerzen. Der Patient gab an, dass bei ihm 2016 bei mäßiger Hyperopie (+3 dpt) eine laserassistierte In-situ-Keratomileusis (LASIK) mit Mikrokeratom beidseits durchgeführt wurde. Er gab sonst keine Augen- oder allgemeine Vorerkrankungen an und nimmt keine systemische Medikation ein.

\section{Klinischer Befund}

Der bestkorrigierte Visus betrug bei Erstvorstellung 0,4 am betroffenen linken $\mathrm{Au}$ ge (subjektive Refraktion: +2,00/-1,50/A $175^{\circ}$ ) sowie 0,8 am rechten Partnerauge (subjektive Refraktion: +1,75/-1,00/A $\left.9^{\circ}\right)$. Spaltlampenbiomikroskopisch zeigte sich rechts ein regelrechter Befund und ein LASIK-Flap mit klarem Interface.
Links zeigten sich eine inselartige Ansammlung von Zellen unter dem LASIKFlap vom nasalen Flaprand bis zur optischen Achse sowie eine parazentrale Lentikelfalte des LASIK-Flaps (• Abb. 1). Der Flap-Hinge war nasal anliegend, die Vorderkammer tief und reizfrei, die Iris intakt und die Linse klar. Fundoskopisch zeigte sich ein regelrechter altersentsprechender Befund beidseits.

\section{Diagnostik}

In der optischen Kohärenztomographie des vorderen Augenabschnitts (VAOCT) beobachteten wir am linken Auge bei ca. $180 \mu \mathrm{m}$ Tiefe eine hyperreflektive, gut definierte Struktur (• Abb. 2).
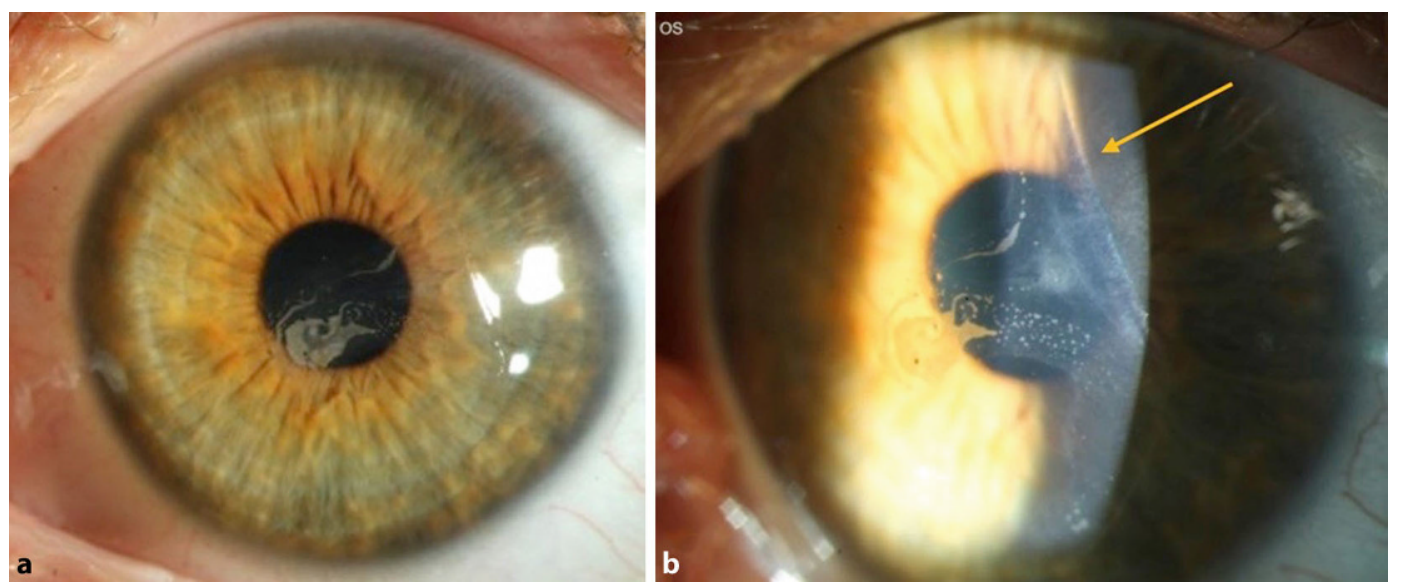

Abb. $1 \varangle$ Klinischer Befund OS. a Überblick. b Lentikelfalte (Pfeil) 


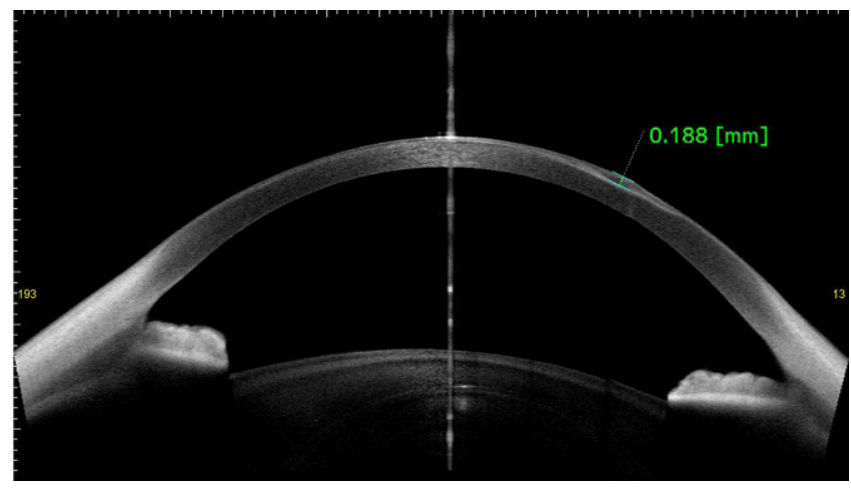

Abb. $2<$ Vorderaugenabschnitts-OCT: hyperreflektive, gut begrenzte Struktur unten dem LASIK-Flap $(188 \mu$ m tief $)$

\section{Wie lautet Ihre Diagnose?}

\section{Definition}

Die Epithelinvasion ist eine seltene postoperative Komplikation bei Zustand nach LASIK. Diese wird in der Literatur zwischen 0 und 3,9\% der Patienten postoperativ angegeben $[2,6]$. Die Pathogenese ist umstritten. Die beiden akzeptierten Hypothesen sind eine intraoperative mechanische Übertragung von Epithelzellen oder eine postoperative Invasion vom Rand des LASIK-Flaps [7]. Während zahlreiche Risikofaktoren für spontane Epithelinvasion beschrieben sind (u.a. Hyperopiekorrektur, Alter des Patienten, Diabetes mellitus Typ 1, Zustand nach operativer Revision wie "flap repositioning“ oder „flap lifting“) [6], bleibt da- neben die posttraumatische Epithelinvasion unter dem LASIK-Flap als häufige Ursache.

\section{\) Diagnose: Posttraumatische LASIK-Flap-Einfaltung mit Epithelinvasion (Grad 4 nach Probst/Machat)}

Die Epithelinvasion wurde 1996 von Probst und Machat in 4 Grade klassifiziert [4] und vor Kurzem von Neff und Probst revidiert [3]: Grad 1: dünn, nicht progressiv, nahe am Flaprand, ohne Progression und ohne Therapiebedarf; Grad 2: dünn, langsam progressiv,

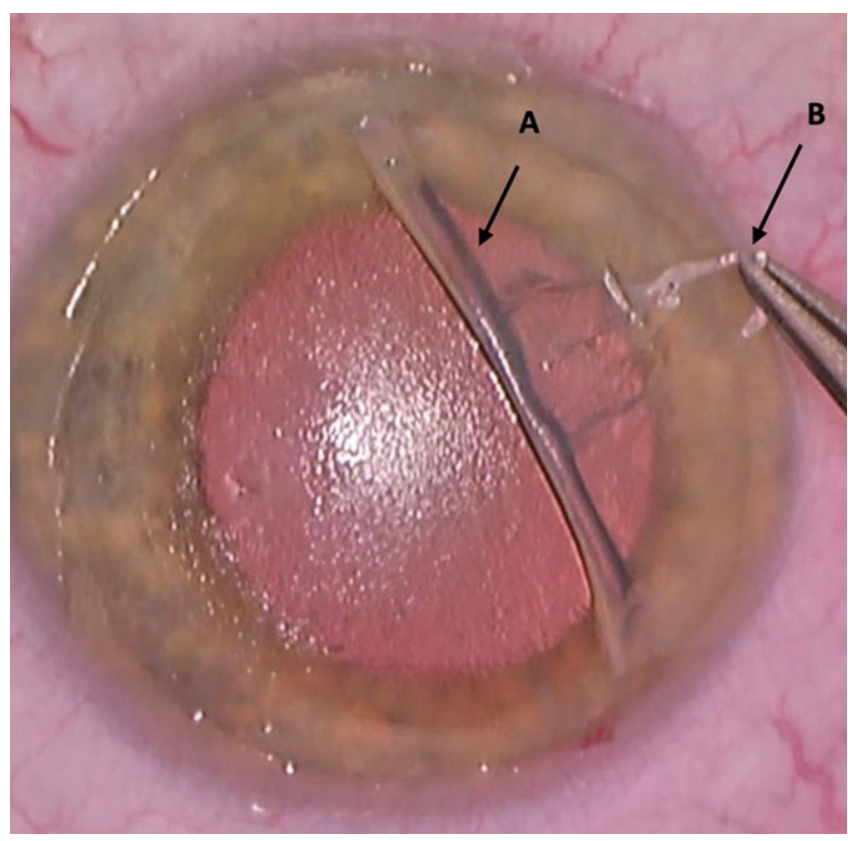

Abb. $3<$ Intraoperatives Bild: Umklappen des Flaps nach nasal (Hinge). $A$ Rückfläche des Flaps (Pfeil). BEntfernen der Epithelinvasion mittels Dreieckstupfern und Knüpfpinzette von der Rückfläche des Flaps (Pfeil). Die intraoperative Mydriasis hilft durch den Rotreflex des Fundus, auch geringste Spuren von Epithel zu identifizieren und zu entfernen nahe am Flaprand, mit nicht dringendem Bedarf einer operativen Therapie; Grad 3 und Grad 4: dicke, undurchsichtige, schnell progressive Epithelinvasion, mehr als $2 \mathrm{~mm}$ vom Flaprand entfernt für Grad 3 oder die optische Achse betreffend für Grad 4. Die Grade 3 und 4 benötigen eine dringende operative Versorgung. Differenzialdiagnostisch sollte eine diffuse lamelläre Keratitis (DLK) (sog. "Sand of Sahara“ [SOS]) ausgeschlossen werden. Diese zeigt sich spaltlampenbiomikroskopisch als Infiltrat unter dem LASIK-Flap mit unterschiedlicher Ausprägung und Ausdehnung je nach Stadium. Stadieneinteilung und Therapie wurden von Bühren et al. genau beschrieben [1].

\section{Therapie und Verlauf}

Wir leiteten am Tag der Diagnose die Therapie mit Prednisolonacetat $10 \mathrm{mg} / \mathrm{ml}$ Augentropfen (AT) stündlich ein und planten für den nächsten Tag eine LASIKFlapanhebung mit mechanischer Exzision der epithelialen Zellen vom stromalen Bett sowie von der Rückfläche des Flaps (- Abb. 3), Flapspülung (mit „balanced salt solution" [BSS], anschließend mit konservierungsfreien Antibiotika), Applikation von $0,02 \%$ Mitomycin C (MMC) als Schwämmchen auf Bett und Flaprückseite während $60 \mathrm{~s}$ und Anlegen von 3 Einzelknüpfnähten an den zurückgeklappten Flapränden (für 4 Wochen [• Abb. 4a]). Die Kombination von mechanischer Abtragung und Applikation von MMC wurde von Wilde et al. als sicher, mit geringer 

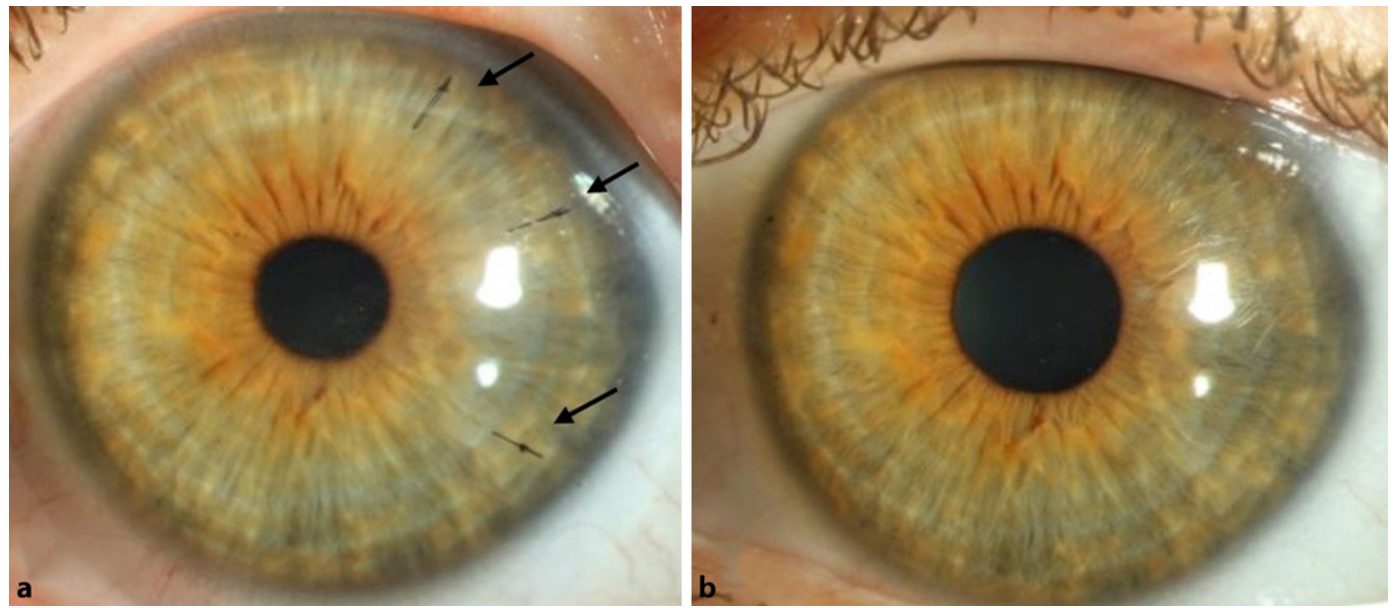

Abb. $4<$ Postoperative Bilder. a Nach 4 Woche: drei 10-0-Nylon-Fäden zur Stellung des Rands (Pfeile). b Nach 4 Monaten: klare Hornhaut, ohne Hinweis auf Rezidiv

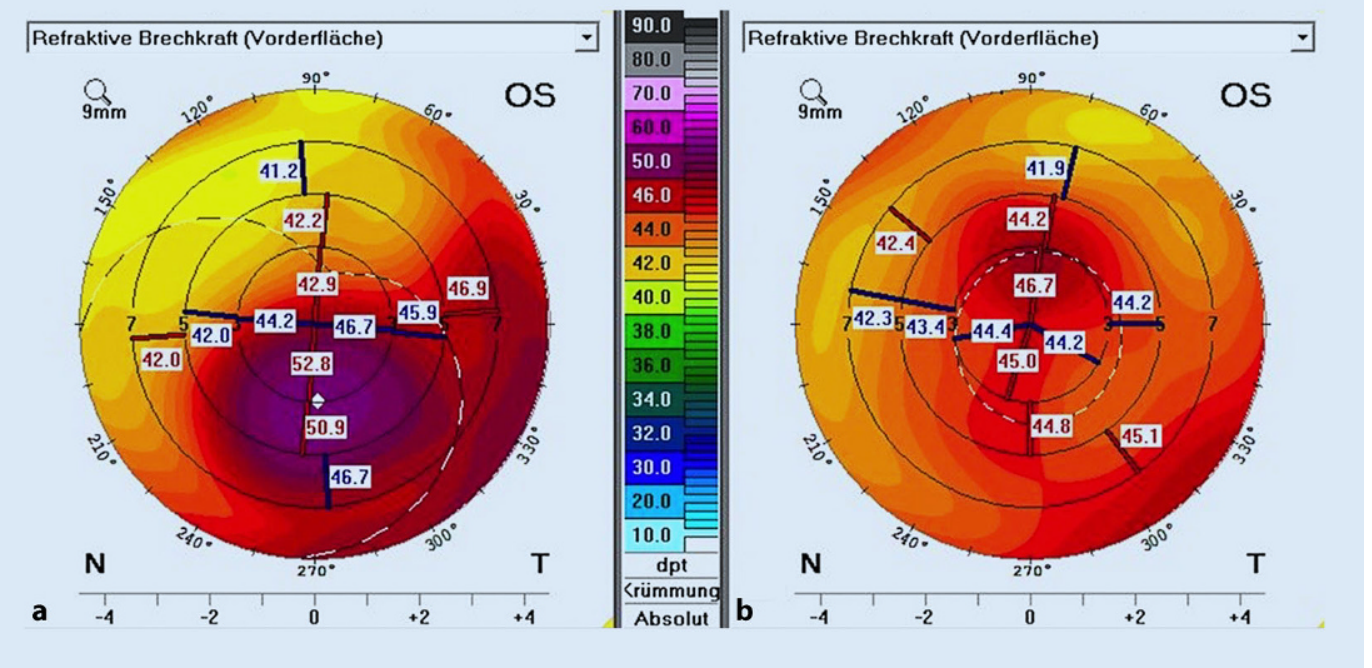

Abb. $5<$ Topographie der Hornhaut: Vergleich zwischen prä- (a) und 4 Monaten postoperativ (b)

Rezidivrate und mit einer guten Visusrehabilitation dargestellt [8]. Bei nicht ausreichender mechanischer Abtragung des Epithels mittels Instrumenten bleibt die Option einer zusätzlichen Excimerlaser-phototherapeutischen Keratektomie (Excimer-PTK) von Bett und Flaprückfläche [5]. Als lokale Therapie bekam der Patient postoperativ Moxifloxacin AT 5-mal/Tag für 1 Woche und Prednisolonacetat $10 \mathrm{mg} / \mathrm{ml}$ AT 5-mal/Tag für 4 Wochen. Bei der letzten postoperativen Kontrolle - 4 Monaten nach der Operation - zeigte sich ein regelrechter und komplikationsloser Befund ohne Hinweis auf Rezidiv (• Abb. 4b). Der Visus betrug 1,0 am betroffenen Auge (sine correctione). Topografisch zeigte sich eine deutliche Regularisierung nach dem Eingriff (• Abb. 5). Die weiteren
Kontrollen wurden durch den niedergelassenen Augenarzt durchgeführt.

\section{Fazit für die Praxis}

- Eine ausführliche Anamnese spielt bei unklarem Hornhautbefund eine entscheidende Rolle für die Diagnosestellung und Therapie.

- Der Begriff „Hornhauttrübung” sollte als Befund oder gar Diagnose vermieden werden.

- Das VA-OCT kann helfen, die Tiefe der Pathologie exakt zu bezeichnen.

- Bei progressiver oder die optische Achse betreffender Epithelinvasion ist eine dringende operative Intervention, unabhängig vom Grad der Visusminderung, indiziert.

- Die mechanische Entfernung des Epithels von Bett und Flaprückfläche in
Kombination mit 0,02\% Mitomycin C für $60 \mathrm{~s}$ scheint derzeit die Methode erster Wahl zu sein.

- Eine intraoperative Mydriasis kann dem Operateur helfen, durch den Rotreflex des Fundus auch geringste Spuren von Epithel zu identifizieren und zu entfernen.

\section{Korrespondenzadresse

Loïc Hamon
Klinik für Augenheilkunde,
Universitätsklinikum des
Saarlandes (UKS)
Kirrbergerstr. 100,
Gebäude 22,66421 Homburg/
Saar, Deutschland
loic.hamon@uks.eu

Funding. Open Access funding provided by Projekt DEAL. 


\section{Einhaltung ethischer Richtlinien}

Interessenkonflikt. L. Hamon, E. Flockerzi, N. Ardjomand, B. Seitz und L. Daas geben an, dass kein Interessenkonflikt besteht.

Für diesen Beitrag wurden von den Autoren keine Studien an Menschen oder Tieren durchgeführt. Für die aufgeführten Studien gelten die jeweils dort angegebenen ethischen Richtlinien. Für Bildmaterial oder anderweitige Angaben innerhalb des Manuskripts, über die Patienten zu identifizieren sind, liegt von innen und/oder ihren gesetzlichen Vertretern eine schriftliche Einwilligung vor.

Open Access Dieser Artikel wird unter der Creative Commons Namensnennung 4.0 International Lizenz veröffentlicht, welche die Nutzung, Vervielfältigung, Bearbeitung, Verbreitung und Wiedergabe in jeglichem Medium und Format erlaubt, sofern Sie den/die ursprünglichen Autor(en) und die Quelle ordnungsgemäß nennen, einen Link zur Creative Commons Lizenz beifügen und angeben, ob Änderungen vorgenommen wurden.

Die in diesem Artikel enthaltenen Bilder und sonstiges Drittmaterial unterliegen ebenfalls der genannten Creative Commons Lizenz, sofern sich aus der Abbildungslegende nichts anderes ergibt. Sofern das betreffende Material nicht unter der genannten Creative Commons Lizenz steht und die betreffende Handlung nicht nach gesetzlichen Vorschriften erlaubt ist, ist für die oben aufgeführten Weiterverwendungen des Materials die Einwilligung des jeweiligen Rechteinhabers einzuholen.

Weitere Details zur Lizenz entnehmen Sie bitte der Lizenzinformation auf http://creativecommons.org/ licenses/by/4.0/deed.de.

\section{Literatur}

1. Bühren J, Cichocki M, Baumeister M, Kohnen T (2002) Diffuse lamelläre Keratitis (DLK) nach Laser-in-situ-Keratomileusis: Klinische und konfokalmikroskopische Befunde. Ophthalmologe 99:176-180

2. Kohnen T, Klaproth OK, Derhartunian V, Kook D (2010) Ergebnisse von 308 konsekutiven Femtosekundenlaserschnitten für die LASIK. Ophthalmologe 107:439-445

3. Neff KD, Probst LE (2011) LASIK complications. In: Krachmer JH, Mannis MJ, Holland EJ (Hrsg) Cornea: surgery of the cornea and conjunctiva. Mosby, St. Louis, MO, S 1861-1882

4. Probst LE, Machat JJ (1996) Corneal subepithelial infiltrates following photorefractive keratectomy. J Cataract Refract Surg 22:281

5. Seitz B, Flockerzi E, Daas L (2020) Bedeutung der Bildgebung pathologischer nicht entzündlicher Befunde der Hornhautoberfläche für die phototherapeutischeKeratektomie.ZPrakt Augenheilkd 41:125-134

6. Ting DSJ, Srinivasan S, Danjoux JP (2018) Epithelial ingrowth following laser in situ keratomileusis (LASIK): prevalence, risk factors, management and visual outcomes. BMJ Open 29:3
7. Wang MY, Maloney RK (2000) Epithelial ingrowth after laser in situ keratomileusis. Am J Ophthalmol 129:746-751

8. Wilde C, Messina M, Dua HS (2017) Management of recurrent epithelial ingrowth following laser in situ keratomileusis with mechanical debridement, alcohol, mitomycin-C, and fibrin glue. J Cataract Refract Surg 43:980-984

\section{Lesen Sie Ihre Zeitschrift online auf SpringerMedizin.de}

SpringerMedizin.de bietet Ihnen Zugang zu allen elektronisch verfügbaren Ausgaben und dem CME-Angebot Ihrer Zeitschrift - unabhängig davon, seit wann Sie die Zeitschrift abonniert haben.

So einfach erhalten Sie Zugang zum Online-Archiv:

- Registrieren Sie sich einmalig auf www.springermedizin.de/register Geben Sie dabei Ihre Einheitliche Fortbildungsnummer (EFN) an.

- Ihr Benutzername entspricht Ihrer E-Mail-Adresse, Ihr Passwort können Sie frei wählen und später jederzeit unter "Mein Profil" ändern.

- Falls Sie bereits ein (Print-) Abonnement bei uns haben, geben Sie bei der Registrierung die Lieferadresse Ihrer Zeitschrift an. Damit wird Ihr Abo-Zugang auf springermedizin.de freigeschaltet.

Sind Sie bereits bei SpringerMedizin.de registriert?

Dann wird Ihr Zeitschriftenabonnement automatisch Ihrem Online-Nutzerkonto

hinzugefügt. Sollten die Angaben Ihres Online-Accounts nicht eindeutig mit den Angaben Ihres Zeitschriften-Abonnements übereinstimmen, kann die Zuordnung nicht sicher erfolgen. In diesem Fall und bei allen anderen Fragen zum Online-Zugang kontaktieren Sie bitte unseren Kundenservice unter: Kundenservice@springermedizin.de 